22. McENIFF, N.; DOHERTY, F.; KATZ, J. y cols.: "Yolk sac tumor of the testis discovered on a routine annual sonogram in a boy with testicular microlithiasis". AJR Am. J. Roentgenol., 164: 971, 1995.

23. HOLM, M.; HOEI-HANSEN, C.E.; RAJPERT-DE MEYTS, E. y cols.: "Increased risk of carcinoma in situ in patients with testicular germ cell cancer with ultrasonic microlithiasis in the contralateral testicle". J. Urol., 170: 1163, 2003.

24. DE GOUVEIA BRAZAO, C.A.; PIERIK, F.H.; OOSTERHUIS, J.W. y cols.: "Bilateral testicular microlithiasis predicts the presence of the precursor of testicular germ cell tumors in subfertile men". J. Urol., 171: 158, 2004.

25. WITTENBERG, K.H.; HATTERY, R.R.; GORMAN, B. y cols.: "Testicular microlithiasis and testicular tumours: association or pre-malignant condition". Radiology, 217: 366, 2000.

26. WEGNER, H.E.; HAMM, B.; LOY, V. y cols.: "Testicular micro-lithiasis: case report and discussion of management under special consideration of germ cell tumours". Int. Urol. Nephrol., 28: 533, 1996.

27. RASHID, H.H.; COS, L.R.; WEINBERG, E. y cols.: "Testicular microlithiasis: a review and its association with testicular cancer". Urol. Oncol., 22: 285, 2004.
Casos Clínicos

Arch. Esp. Urol., 59, 1 (81-84), 2006

\section{LEIOMIOMA RENAL: APORTACIÓN DE UN NUEVO CASO.}

Angel Andreu García, Enrique Herrero Polo, José Miguel Alapont Alacreu, Mํa Belén Coronel Sánchez, Enrique Schiefenbuch Munné, Roberto Botella Almodovar, José Luis Brotons Márquez y Gonzalo Llamazares Cachá.

Servicio de Urología. Hospital Virgen de la Salud de Elda. Elda. Alicante. España.

Resumen.- OBJETIVO: Aportar un nuevo caso de leiomioma capsular asintomático.

MÉTODO: Paciente de 68 años asintomático que tras estudio radiológico (ECO y TAC) es diagnosticada de masa sólida.

RESULTADO: El tratamiento de este proceso neoformativo renal, fue nefrectomía radical, con curso postoperatorio sin incidencias y anatomía patológica, leiomioma renal.

CONCLUSIÓN: El leiomioma renal es un fumor mesenquimatoso benigno poco frecuente que debe tenerse en cuenta ante el diagnóstico de masa renal, dado que sería subsidiario de una cirugía renal conservadora, no obstante ante la dificultad para diferenciarlo del carcinoma renal, no es infrecuente que el diagnóstico se produzca tras el análisis histológico de la pieza de nefrectomía.

Palabras clave: Leiomioma. Cápsula renal. Tumores renales benignos. 
Summary.- OBJECTIVES: To report a new case of asymptomatic capsular leiomyoma.

METHODS: 68-year-old male asymptomatic patient who was diagnosed of solid mass after a radiological study (ultrasound and CT scan).

RESULTS: The treatment of this neoplasia was radical nephrectomy, with an uneventful postoperative course and a pathology report of renal leiomyoma.

CONCLUSIONS: Renal leiomyoma is a rare benign mesenchymal tumor that should be taken into consideration for the differential diagnosis of renal masses, because it would be an indication for nephron-sparing surgery; nevertheless, due to the difficulties to differentiate it from renal cell carcinoma it is not rare that the diagnosis is achieved after histological study of the nephrectomy specimen.

Keywords: Leiomyoma. Renal capsule. Benign renal tumors.

\section{INTRODUCCIÓN}

Los leiomiomas se incluyen dentro del grupo de los tumores benignos derivados del tejido conjuntivo o mesenquimatoso, y pueden implicar a cualquier estructura del aparato genitourinario que presente músculo liso entre sus componentes histológicos $(1,2)$. Son tumores poco frecuentes clínicamente, aunque en estudios de autopsia son el nódulo renal benigno mas frecuente, después de los adenomas $(3,5-7)$.

Debido a su excepcional incidencia no suelen ser incluidos en el diagnóstico diferencial de masa renal, y ayudado por la falta de especificidad de la semiologia iconográfica, nos lleva en la mayoría de ocasiones a realizar una cirugía radical en una lesión benigna, cuando podría ser subsidiaria de realizar una cirugía conservadora.

\section{CASO CLÍNICO}

Paciente de 68 años de edad, con antecedentes de obesidad, brucelosis, lumboartrosis, litiasis biliar, apendicectomía y cesárea.

En estudio ecográfico de la vía biliar, se objetiva de forma casual, masa de $6 \mathrm{~cm}$. de diámetro en polo superior de riñón derecho. La TAC confirma este hallazgo evidenciándose masa sólida en polo superior de riñón derecho; comprobándose realce de la misma tras la infusión de contraste, no objetivándose adenopatías hiliares, ni afectación de órganos de vecindad (Figura 1). Con el diagnóstico de masa sólida compatible con adenocarcinoma renal es intervenida, realizándose por vía lumbar oblícua nefrectomía radical, siendo el curso postoperatorio sin incidencias.
En el estudio microscópico se objetiva tumoración en polo superior que no invade cápsula, su aspecto al corte es homogéneo, de color blancogrisaceo. La pelvis y cálices no están afectados por la tumoración y la vena renal esta libre.

En el estudio microscópico se observa tumoración redondeada de márgenes bien definidos, que crece por expansión sin invadir, de naturaleza mesenquimal, compuesta por células dispuestas en fascículos entrelazados, los núcleos presentan ligera variación de tamaño y forma, la actividad mitótica es inferior a 5 mitosis por 50 CGA, no se aprecian hemorragias ni necrosis (Figura 2).

En el estudio inmunohistoquímico se objetiva negatividad para queratinas (AE-1 AE-3), EMA (anfígeno epitelial de membrana) con lo que se excluye la naturaleza epitelial del tumor. Negatividad para vimentina, HMB45, S-100. Positividad para marcadores vasculares en la trama vascular y positividad para actino.

\section{DISCUSIÓN}

El leiomioma se incluye dentro del grupo de los tumores benignos derivados del tejido conjuntivo o mesenquimatoso. Son poco frecuentes y pueden darse en cualquier órgano del aparato genitourinario que contenga músculo liso se ha estimado que el $90 \%$ de los leiomiomas renales se originan en la cápsula renal y el $10 \%$ restante en el músculo liso de la pelvis renal $(3,8)$. Son mas frecuentes en mujeres $(66 \%)$, siendo mas común entre la $2^{\mathrm{a}}$ y $5^{\mathrm{a}}$ década de la vida. La manifestación clínica mas frecuente es la presencia de una masa abdominal palpable $(57 \%)$, asociada o no a dolor. A veces hay hematuria, aunque la tríada clásica de Guyón solo esta presente en el $3,3 \%$ de las ocasiones $(5,6)$.

La escasa frecuencia de estos tumores renales no ha permitido describir unos patrones radiológicos específicos, lo que ha llevado a realizar cirugías radicales en nume-

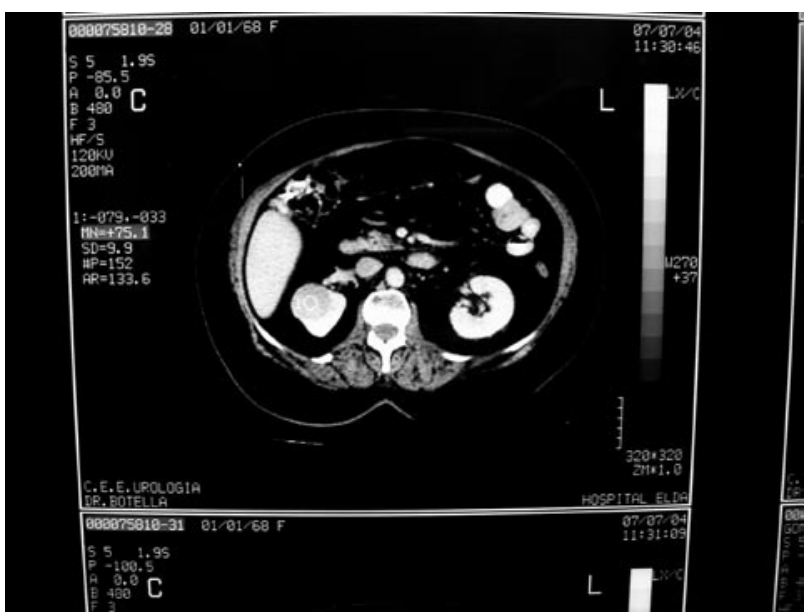

FIGURA 1. Masa en polo superior de riñón derecho. 
rosas ocasiones, ante el diagnóstico de hipernefroma, ya que frecuentemente es indistinguible radiologicamente del mismo (ECO, TAC, RMN).

Algunos autores indican algunas características que si bien no son específicas, pueden hacer sospechar la existencia de este tipo de tumores (5):

- Patrón sonográfico algo menos refringente que el resto del parénquima renal.

- Lesión bien circunscrita (hipo o hipervascularizada), no presentando invasión de la vena renal, adenopatías, ni invasión de órganos vecinos.

- Ausencia de calcificaciones, aunque aparecen en el $20 \%$ de los casos.

La TAC es mas sensible que la ECO para valorar la presencia de la lesión, objetivando masas bien circunscritas, de predominio periférico capsular o peripiélico, de naturaleza sólida, con densidad de partes blandas en los leiomiomas debido al componente predominante de músculo liso reflejarían densidades de tejidos blandos (45 $\mathrm{UH})$, inferiores a los registrados en los hipernefromas $(60-80 \mathrm{UH}),(6,7)$.

La RNM ofrece información similar a la TAC, definiendo lesiones sólidas con adecuado plano de clivaje entre el tumor y el parénquima renal $(5,6)$.

El perfil inmunohistoquímico de los leiomiomas renales revela marcadores de músculo liso, así estos tumores expresan negatividad a las citoqueratinas de bajo peso molecular (BDK, AE 1) y positividad frente a vimentina, actina y desmina (6).

La positividad al HMB-45 se ha descrito fundamentalmente en tumores de origen hamartomatoso como el angiomiolipoma, rabdomioma y linfangioma. En nuestro caso fue absolutamente negativo, excluyéndolo de cualquier origen hamartomatoso. (5)

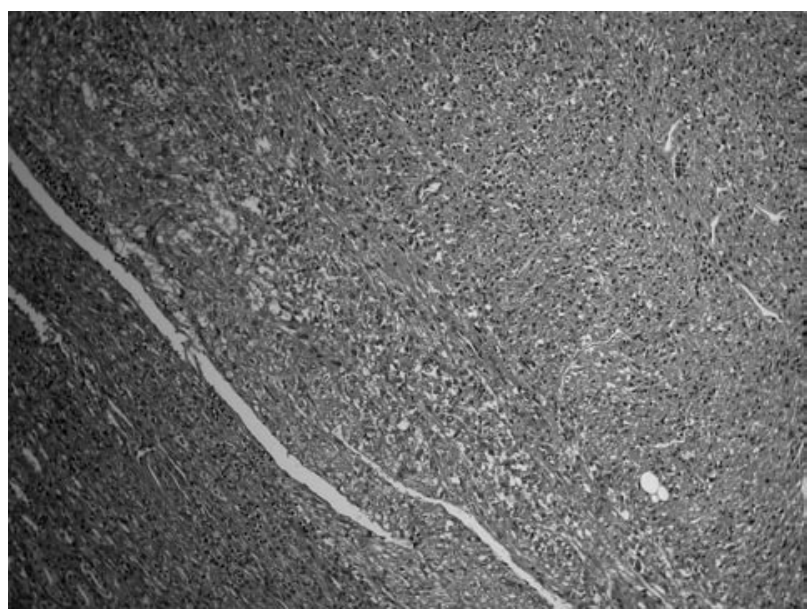

FIGURA 2. Tumoración mesenquimal compuesta por células dispuestas en fascículos entrelazados.
Es conocida la asociación entre el virus de Epstein-Barr y la presencia de tumores en pacientes inmunodeprimidos, entre ellos los de músculo liso, por ello el leiomioma debe tenerse en cuenta a la hora de plantear el diagnóstico diferencial de las masas renales en este subgrupo de población. (5)

De forma general siempre se debe pensar en este tipo de tumor dado su comportamiento benigno (4), ante una masa con las características radiológicas descritas anteriormente, dado que la cirugía preservadora de nefronas es una alternativa factible a la nefrectomía (9).

El pronóstico de este tipo de tumor es excelente, si bien excepcionalmente se ha constatado la metastatización, que al igual que el tumor primario conserva su aspecto histológico benigno (9).

A modo de conclusión, el leiomioma renal es una tumoración infrecuente que debe incluirse en el diagnóstico diferencial de las masas renales, sobretodo en mujer joven asintomática con masa voluminosa en riñón, de predominio periférico capsular o peripiélico, de naturaleza sólida, con densidad de partes blandas y en ausencia de invasión de órganos de vecindad, sin afectación ganglionar, visceral ni sistémica.

No obstante ante la imposibilidad de un diagnóstico preoperatorio de certeza, se aconseja la exéresis radical como primera opción (10).

\section{BIBLIOGRAFÍA y LECTURAS RECOMENDADAS ( ${ }^{*}$ lectura de interés $y^{* *}$ lectura fundamental)}

1. NAGAR, A.M.; RAUT, A.A.; NARLAWAR, RS. y cols.: "Giant renal capsular leiomyoma: study of two cases”. Br. J. Radiol., 77: 957, 2004.

2. RABADA REY, C.J.; FERNANDEZ GOMEZ, J.M.; ALVAREZ FONSECA, S. y cols.: "Leiomioma renal. Aportación de un nuevo caso". Actas Urol. Esp., 18: 816, 1994.

3. RISQUET, H.; FABRE-BOCQUENTIN, B.; SKOVRON, O. y cols.: "Report of a case of leiomyoma of the kidney". Prog. Urol., 12: 92, 2002.

4. PROTZEL, C.; WOENCKHAUS, C.; ZIMMERMANN, U. y cols.: "Leiomyoma of the kidney. Differential diagnosis aspects of cell carcinoma with increasing clinical relevance". Urologe A., 40: 384, 2001.

**5. CLEMENTE RAMOS, C.M.; CANDIL FERNANDEZ, A.; ALLONA ALMAGRO, A.: "Leiomioma renal sintomático una masa renal de difícil diagnostico". Actas Urol. Esp., 27: 546, 2003.

**6. PEREIRA ARIAS, J.G.; ULLATE JAIME, V.; GUTIERREZ DIEZ, J.M. y cols.: "Leiomioma renal voluminoso”. Actas Urol. Esp., 25: 81, 2001.

7. STEINER, M.; QUILAN, D.; GOLDAM, S.M. y cols.: "Leiomyoma of the kidney: presentation of 
4 new cases and the of computerized tomography". J.Urol., 43: 994, 1990.

8. MONTOYA LIROLA, M ${ }^{\mathrm{a}}$.D.; GARCIA TABAR, P.J.; GUTIERREZ DIEZ, J.M. y cols.: "El leiomioma renal sintomático". Arch. Esp. Urol., 46: 833, 1993.

*9. RAMÓN CORTADELLAS, A.; CASTELLANOS ACOSTA, R.I.; GUZMÁN FERNÁNDEZ, A. y cols.: "Leiomioma de cápsula renal.Presentación de un caso y revisión de la literatura". Arch. Esp. Urol., 45: 478, 1992.

10. LLAMAZARES CACHA, G.; IBARZ SERVIO, L.: "Leiomioma renal del adulto". Actas Urol. Esp., 4: 269, 1980.

Casos Clínicos

Arch. Esp. Urol., 59, 1 (84-87), 2006

\section{COLISIÓN TUMORAL EN HIDRONEFROSIS GIGANTE.}

José Gregorio Pereira Arias, Vicente Ullate Jaime, José María Gutiérrez Díez, Ricardo Ateca Díaz-Obregón, María Mar Ramírez Rodríguez', Esther Pereda Martínez? y José Ramón Berreteaga Gallastegui.

Servicio de Urología. Sección de Antomía Patológica' Hospital de San Eloy. Baracaldo. Vizcaya. España.

Resumen.- OBJETIVO: La hidronefrosis gigante, definida por la presencia de un volumen de líquido superior a los 1000 mililitros en el interior del sistema colector urinario, es una realidad clínica infrecuente cuyo diagnóstico resulta un excelente ejercicio al carecer de una personalidad clínica definida.

MÉTODO Y RESULTADOS: Presentamos un paciente de 66 años de edad, en estudio por sospecha de tumoración digestiva al presentar un cuadro de larga evolución, caracte-

\section{J.G. Pereira Arias}

Servicio de Urología. Hospital San Eloy.

Avenida Antonio Miranda,5

Baracaldo 48902. Vizcaya. (España)

urología.saneloy@hsel.osakidetza.net

Trabajo recibido: 5 de mayo 2005 rizado por caquexia severa, astenia, anemia, estreñimiento y masa abdominal. Las pruebas de imagen constataron una gran hidronefrosis derecha secundaria a una litiasis en la unión pieloureteral. Se practicó nefrectomía simple, evacuando $7.800 \mathrm{ml}$ de contenido serohemático del interior del sistema pielocalicial. El diagnóstico histológico reveló la presencia de múltiples focos de carcinoma transicional y carcinoma de células claras asociado. Revisamos aspectos diagnósticos y terapéuticos en la literatura.

CONCLUSIONES: La hidronefrosis gigante representa un dilema diagnóstico pudiendo presentarse de forma asintomática, condicionar clínica derivada de la compresión de estructuras abdominales (obstrucción intestinal o urinaria), o bien simular fumores abdominales, ascitis masiva o lesiones quísticas retroperitoneales. El tratamiento de elección, en la mayoría de ocasiones, es la nefrectomía simple debido al avanzado deterioro de la unidad renal implicada. No obstante, en ocasiones y en pacientes comprometidos es necesario el drenaje percutáneo previo o aislado para evitar cambios en el equilibrio hemodinámico secundarios a la súbita descompresión abdominal.

Palabras clave: Hidronefrosis gigante.

Summary.- OBJECTIVES: Giant hydronephrosis, defined as the presence of a liquid volume over $1000 \mathrm{~m} /$ within the urinary collector system, is a rare clinical entity, the diagnosis of which is an excellent exercise because it lacks of a defined clinical presentation.

METHODS/RESULTS: We report the case of a 66-year-old male under study for a suspicious digestive tumor due to a long lasting clinical picture with severe cachexia, asthenia, anemia, constipation, and abdominal mass. Radiological tests showed a great right hydronephrosis secondary to a culculus in the ureteral-pelvic junction. Simple nephrectomy was performed, evacuating $7800 \mathrm{ml}$ of serous-hematic liquid from the pyelocalicial system. A histological diagnosis revealed the presence of multiple foci of transitional cell carcinoma and renal cell carcinoma associated. We review the diagnostic and therapeutic features in the literature.

CONCLUSIONS: Giant hydronephrosis represents a diagnostic dilemma. It may present as an asymptomatic process, with clinical features of abdominal organs compression (bowel or urinary obstruction) or simulate abdominal tumors, massive ascites, or cystic retroperitoneal lesions. Simple nephrectomy is the treatment of choice in most cases, due to the advanced deterioration of the renal unit. Nevertheless, in some cases, in compromised patients, percutaneous drainage may be necessary as previous or definitive treatment to avoid changes in the hemodynamic balance secondary to the sudden abdominal decompression.

Keywords: Giant hydronephrosis. 